\title{
BreastSimulator: A software platform for breast $x$-ray imaging research
}

\author{
Kristina Bliznakova ${ }^{1}$, I oannis Sechopoulos ${ }^{2}$, Ivan Buliev ${ }^{3}$, Nicolas Pallikarakis ${ }^{1}$ \\ 1. Department of Medical Physics, School of Medicine, University of Patras, Greece. 2. Department of Radiology and \\ Imaging Sciences and Winship Cancer Institute, Emory University School of Medicine, Atlanta, Georgia. 3. Department of \\ Electronic Engineering and Microelectronics, Technical University of Varna, Varna, Bulgaria.
}

Correspondence: Nicolas Pallikarakis. Address: Department of Medical Physics, School of Medicine, University of Patras 26500, Rio, Patras, Greece. Telephone: 30-261-099-7781. Fax: 30-261-099-2496. Email: nipa@upatras.gr.

Received: February 24, 2012

DOI : $10.5430 / j b g c . v 2 n 1 p 1$
Accepted: March 21, $2012 \quad$ Published: June 1, 2012

URL: http://dx.doi.org/10.5430/jbgc.v2n1p1

\section{Abstract}

Objective: It is well established that computer based models of x-ray imaging systems are basic and very important tools for developing and evaluating new emerging $\mathrm{x}$-ray imaging techniques, optimizing technical parameters, and performing feasibility studies prior to implementation in clinical practice. Such models are essential for the development and the establishment of new breast x-ray imaging modalities that aim to detect and better characterize breast lesions in their early stage. This work presents a complete software package, called BreastSimulator, dedicated for breast x-ray imaging research.

Methods: The package consists of four modules used to create three-dimensional breast models in compressed and uncompressed state, simulate $\mathrm{x}$-ray mammographic images and visualize the results of the simulations. The module that is used to generate breast models, Breast Modeling Module, consists of several sub-modules that are utilized to model the different breast components: external shape, glandular and adipose tissue, breast lesion, skin, pectoralis and lymphatics. The Compression Module is dedicated to simulate the mechanical compression of the breasts. Mammographic projection images are obtained with simulation of $\mathrm{x}$-ray photon transport starting from the $\mathrm{x}$-ray source, passing through the breast model and reaching the detector. This is accomplished in the Image Generation Module. Finally, the results of the simulations, i.e. breast models and mammographic images can be seen with the Visualization Module.

Results: Here, we demonstrate the application of the software package in conventional and dual-energy mammography as well as compression studies, as examples to highlight basic functions and applications of Breast Simulator. The first study aimed to define the optimal pair of 'low' and 'high' monochromatic x-ray energies for dual-energy mammography. It involved the synthesis of 225 dual-energy images obtained from combinations of 'low' and 'high' energy images acquired in the energy range 14 to $28 \mathrm{keV}$. Images were generated from a medium sized dense breast model that contained one calcification. The study showed that $17 / 28 \mathrm{keV}$ incident monoenergetic beams are optimal to obtain maximal calcification detectability for this breast. The second study demonstrated the effect of breast compression on the quality of the obtained mammograms. It included a breast model based on breast CT slices subjected to simulated compression and generation of mammographic images. Increased image quality is observed for mammograms obtained from breasts with reduced thickness. The characteristics of the x-ray beams that exit a small dense breast model were investigated in the third study. 
For two mammographic spectra used in mammography imaging, the mean energy of the transmitted x-rays and the mean exit angle of the scattered radiation increase as the incident $x$-ray energy increases.

Conclusions: We believe that this tool and its functionalities will speed up the development, testing and optimization of new breast imaging modalities such as breast tomosynthesis, cone-beam CT and advanced two-dimensional techniques like dual-energy as well as specific parts of imaging chain, such as x-ray source, detector and acquisition geometry.

\section{Key words}

Simulation package, Three-dimensional breast models, Breast compression, Mammography

\section{Introduction}

The most common form of cancer diagnosed in European women remains breast cancer, contributing to almost $4 \%$ of all female deaths ${ }^{[1]}$ and $29 \%$ of all diagnosed cancer cases ${ }^{[2]}$. In Europe, one in ten women develops this type of cancer in her lifetime. As the population ages, there will be an increase in the number of women that will be affected by this disease. Presently, x-ray mammography screening carried out according to EU Guidelines is the best form of early breast cancer detection. Despite technological advances, such as digital mammography, screening and diagnosing cancers hidden in dense breast parenchyma remains a challenging task. The insufficient soft tissue contrast and the effect of overlying structures in x-ray mammograms are the basic causes that result in approximately 10 to $20 \%$ of the palpable breast cancers being missed during screening mammography ${ }^{[3]}$.

The development of digital detector technology led to the introduction of novel advanced x-ray techniques that, applied to breast imaging, may result in improved detection and diagnosis of breast cancer. Such an example is Breast Tomosynthesis (BT) that has the potential to be more accurate, particularly in the case of breast masses in dense breasts and to extract additional diagnostic information in comparison to conventional mammography ${ }^{[4]}$. One commercial BT product has been recently approved by the Food and Drug Administration and released on the market. Two other vendors are in process of developing similar devices. However, system features, acquisition geometries and reconstruction algorithms are still a topic of extensive research, since their choice significantly influences the image quality of BT. Moreover, early clinical experience with BT reveals difficulties in the interpretation of breast tomograms, due to insufficient training and lack of experience of radiologists with this new modality ${ }^{[5]}$. Some other examples of advanced breast techniques include dedicated cone-beam breast $\mathrm{CT}$ and contrast-enhanced $\mathrm{BT}{ }^{[6]}$. These new modalities have recently demonstrated good results and may, in the future, also play a role in detecting and/or diagnosing breast cancer if some aspects of the technology (e.g. detectors, scanning time, etc) are further improved.

Modeling and simulation are important tools, primarily exploited in the development, optimization and the establishment of novel breast imaging techniques for screening and diagnostic imaging. These are instruments commonly used to evaluate both the performance of new modalities in their entirety (e.g. BT or dedicated breast CT) as well as for just specific parts of x-ray imaging systems (e.g. detectors, $x$-ray spectra, or acquisition geometries). The main components of breast imaging simulations are modeling the components of the x-ray imaging system: $\mathrm{x}$-ray spectra, detector, acquisition geometry and the breast itself, and a computer code to simulate the radiation transport in the model, resulting in generation of projection mammography images and evaluation of the dose absorbed in the breast. The precise modeling of all these components will ensure results approaching what would be obtained in practice.

The main output of breast $x$-ray imaging simulations is the two-dimensional (2D) mammographic images, which are then processed according to the aims of each study. Synthetic mammograms are calculated utilizing one of two approaches. The most popular approach is to simulate the beam transport through the mammographic system based upon the exponential attenuation of incident beams ${ }^{[7-10]}$. This approach produces images quickly but is limited to the simulation of primary radiation effects only. In addition, the radiation dose cannot be estimated precisely. Alternatively, Monte Carlo simulation codes ${ }^{[11]}$ that simulate the beam interaction, including the scattered radiation, have been developed. Such 
simulations are system- and application specific ${ }^{[12]}$ and can be used to generate synthetic mammograms that approach reality which can be used to study particular aspects of the imaging chain.

Amongst the main components of an x-ray breast imaging simulation system is the model of the breast. The use of either a simple or complex model can have a large impact on the study results. Simple mathematical breast phantoms, usually in the form of cylinder, half-ellipsoid or slabs, are widely used in simulations particularly for dosimetry and optimization of acquisition geometry ${ }^{[12-15]}$. In most cases, they are represented by a homogeneous material (e.g. polymethyl methacrylate) that combined with the phantom thickness results in a desired glandular to adipose breast ratio. However, due to the homogenous background, their use is limited whenever a real tissue background is required (to investigate the detectability of lesions, the performance of image processing algorithms, the reconstruction algorithms, etc).

Advanced breast models with realistic three-dimensional (3D) breast tissue distribution and anatomical features ${ }^{[8,10,16-18]}$ have also been developed. They offer a flexible and simple way to investigate different aspects of 2D and 3D x-ray breast imaging: to perform accurate breast dosimetry ${ }^{[19]}$, to calculate the properties of the digital mammograms ${ }^{[9]}$ and to investigate the effect of BT acquisition parameters on computer-extracted texture features ${ }^{[20]}$. These models offer complex breast tissue simulation and allow the generation of synthetic mammograms which resemble real ones. However, none of them is complete and perfect yet. Some of them lack particular anatomical features, some models are unrealistic for some applications, the spatial resolution is insufficient, in some cases compression is not applied, and some models cannot be extrapolated towards all new emerging modalities. Breast phantoms with realistic tissue distributions may be also created from segmented clinical data sets acquired by breast $\mathrm{CT}^{[21-23]}$. Such design guarantees a very high degree of realism; however, it represents a single breast composition and lacks flexibility to cover wide anatomical variations.

A methodology for creation of 3D breast models was reported, but with some limitations in breast type, size and resolution ${ }^{[18]}$. Further research in this field led to the development of an algorithm for simulation of compression of soft tissues ${ }^{[24]}$ and an improved version of the methodology for creation of 3D breast phantoms with a projection appearance similar to clinical images ${ }^{[10]}$. In its initial form (based on the publication from 2003), this software has been provided to over 20 research and industry organizations and has been regarded as a useful tool to predict the performance of imaging procedures. In order to respond further to increasing demands from the research community, we further incorporated the novel methodology for breast creation as well as the algorithm for breast compression. This paper reports on the development of the entire Breast Simulator software package dedicated for breast x-ray imaging research. The package consists of four modules used to create 3D breast models, compress them, simulate their x-ray images and finally visualize the results of the simulations, i.e. 3D breast models and 2D projection images. They are outlined in section Materials and Methods. Examples that highlight basic functions and applications of the Breast Simulator package, particularly for dual-energy mammography, compression studies in standard mammography and use of breast models to study energy and angular distributions of $\mathrm{x}$-rays that exit the breast during mammography are reported as results.

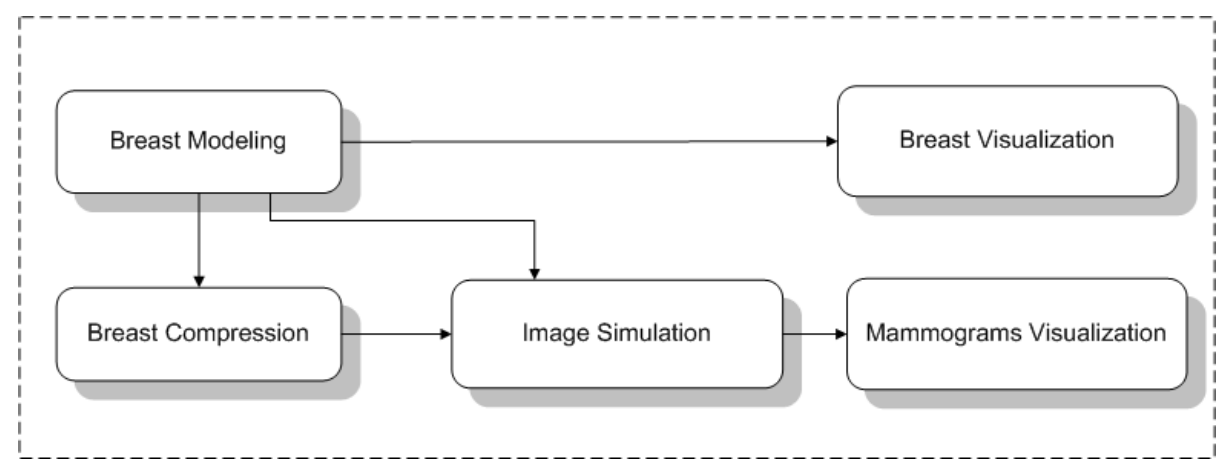

Figure 1. Main breast simulator functionalities. Breast Modeling Module may also produce phantoms from segmented breast CT slices. 


\section{Materials and methods}

\subsection{System description}

A major advantage of the presented software package is its modular architecture. The current software system is comprised of a set of separate modules that address specific modeling issues. Specifically, the system, schematically presented in Figure 1, comprises four basic software modules: (a) Breast Modeling Module - to generate 3D breast models; (b) Breast Compression Module - to compress breast models, (c) Image Generation Module - to model the x-ray transport and generate synthetic projection images and (d) Visualization Module - set of utilities to visualize 2D and 3D breast images.

\subsubsection{Breast modeling module}

This module consists of several sub-modules that are used to model the different breast components: (a) external shape; (b) glandular tissue: ducts and Cooper ligaments; (c) adipose tissue; (d) abnormalities like regular and irregular masses, spherical and ellipsoid calcifications and group of micro calcifications ( $\mu$ Cas), elongated abnormalities; (e) skin, pectoralis and lymphatics. The methodology for generation of realistic 3D breasts and extensive objective and subjective evaluation studies has been recently reported by our group ${ }^{[10]}$. Briefly described, the breast surface is modeled as a combination of two geometrical primitives: an elongated semi-ellipsoid and an elongated semi-hyperboloid. For breast CT and cone-beam CT applications the external shape may be modeled with a single semi-ellipsoid. The duct system is simulated using a network of cylinders, probabilistically arranged in the breast as branches, in a tree-like arrangement, starting from the nipple and restricted by the external breast contour. The mammographic texture simulates the presence of adipose, fibrous and connective tissues as well as other non-glandular tissue types that are not explicitly modeled. The algorithm is based on the use of random walks, calculated using the concept of the 'fractional Brownian motion model', followed by a series of 3D image processing algorithms that result in a realistic 3D texture. Cooper's ligaments are modeled as a set of thin ellipsoid shells, originating at randomly sampled positions in the breast model, while the pectoralis muscle is approximated as a cone shaped object. Breast abnormalities are modeled with round, ovoid, elongated or irregular shapes. Lymphatic system is included as well. The final breast is a 3D matrix or several 3D matrices composed of voxels with resolution and content defined by the user.

To increase the ease of use of the breast model generation module, a graphical user interface application for MS Windows has been created, helping accelerate the parameter input process and providing some visual feedback. Using it, the modeling of the separate breast components is accomplished from a menu shown in Figure 2a. The choice of different items from this menu invokes dedicated dialog forms, each of which comprises several fields that are used to define and select the proper geometrical (dimensions) and physical (attenuation coefficients) parameters of the modeled breast components. An example is depicted in Figure $2 \mathrm{~b}$ showing a screenshot from modeling a cluster of $\mu$ Cas. The geometrical parameters of the cluster are: the radius as well as the number of $\mu$ Cas and their position in the model, while the absorption characteristics are represented by the attenuation coefficient that in this particular example is $1.85 \mathrm{~mm}^{-1}$ corresponding to the attenuation of calcium carbonate for an incident x-ray energy of $19 \mathrm{keV}$. A preview window is available to the user to facilitate the modeling of the external shape, i.e. setting the dimensions (see Figure 2a).

There are several outputs from the breast modeling process: (a) a breast phantom without mammography texture (see Figure 2c); (b) an attenuation coefficients breast model; (c) a model that contains the elemental compositions of simulated breast tissues (Figure 2d), and (d) a mammography texture itself. The second model is useful for generation of mammographic images of the breast using monochromatic incident x-ray beams (e.g. $19 \mathrm{keV}$ ). The third model is exploited with Monte Carlo simulations and breast compression. The breast without mammographic texture is useful for creation of breast models with different backgrounds and the same breast components comprised of 3D geometrical primitives such as the duct system, breast lesions and so on. The texture itself may be used for evaluation of texture characteristics and for creation of models that have the same texture and different breast structure. 


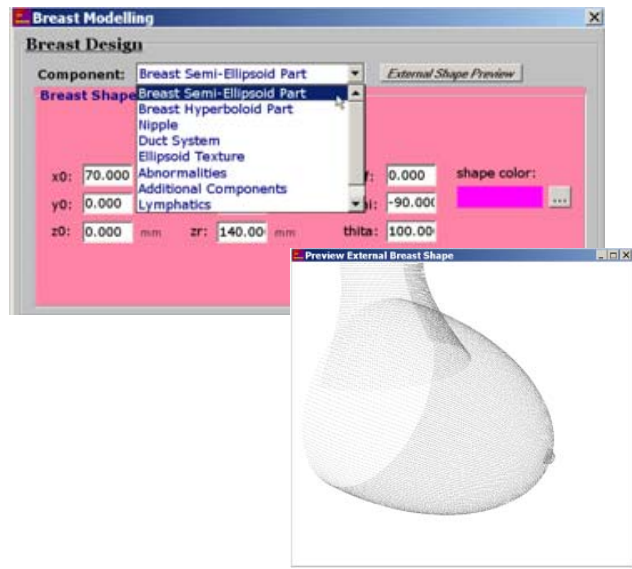

(a)

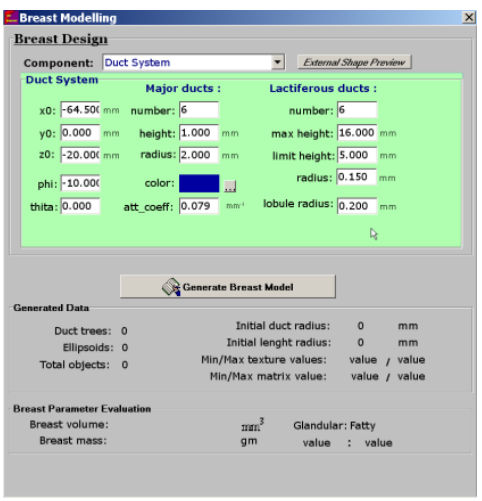

(b)

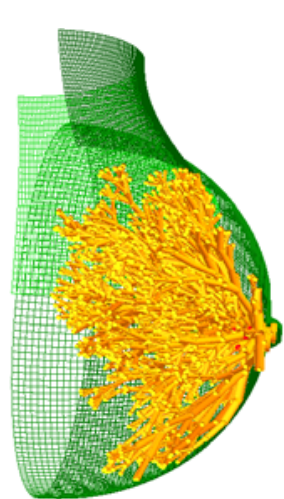

(c)

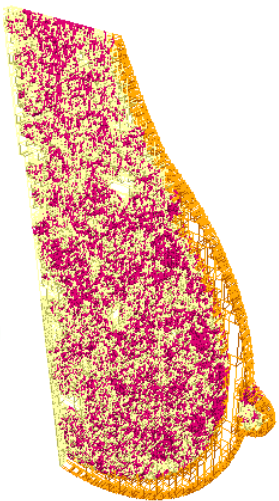

(d)

Figure 2. Screenshots from the software application showing: (a) design of breast components and auxiliary window helpful in setting the external dimensions; (b) design of two clusters of ten microcalcifications each. Main outputs of the breast modeling process: (c) a breast phantom without mammography texture and (d) a composite model that contains the elemental compositions of the simulated breast tissues.

Another approach is the use of external breast models, for example patient specific data obtained from breast CT. In this case, pre-processing of breast data such as denoising of CT slices and tissue segmentation ${ }^{[21-23]}$ is required.

Breast models are saved in a binary format, which can be imported and viewed in other imaging applications.

\subsubsection{Compression module}

The Compression Module is dedicated to simulate the mechanical compression of the breasts. The compression algorithm is a general algorithm for soft tissue compression ${ }^{[24]}$, which is based on a linear spring model. The mechanical properties of the tissues are assumed to be linear and isotropic. The breast volume subjected to compression is divided into a number of "model elements" each one consisting of 27 nodes (voxels). The nodes in the model elements are connected with springs. The spring is defined by two parameters: modulus of elasticity and equilibrium length. The spring modulus of elasticity is assumed to be linear and isotropic, i.e. it is a constant for all the range of deformations that can be applied on the spring. Each node represents a tissue sample and hence it inherits the modulus of elasticity of that tissue. During compression, the volume remains constant due to the introduced concept of spring variable equilibrium lengths.

Simulation of breast compression is applied to the breast models for the purposes of mammography simulation and breast tomosynthesis. Table 1 summarizes the modulus of elasticity of the tissues that are used in the breast compression. To facilitate the specification of the position of the plates and the desired final compressed breast thickness in the compression process, a visualization module has been introduced.

Table 1. Values for Young's modulus

\begin{tabular}{lll}
\hline Tissue & Young's modulus, kPa & Literature \\
\hline Adipose & 1.0 & {$[35]$} \\
Glandular tissue & 10.0 & {$[35]$} \\
Skin & 88.0 & {$[35]$} \\
Calcification & 43.0 & {$[36]$} \\
Cyst & 17.0 & {$[36]$} \\
\hline
\end{tabular}




\subsubsection{X-ray imaging module}

This module contains information for the acquisition geometry (e.g. distances from the source to the object plane and detector), gantry angles, detector type and other imaging parameters. Both monochromatic and polychromatic beams may be simulated. Acquisition of standard mammography, BT, CT, cone-beam and helical CT images of the developed breast model may be modeled and simulated. The x-ray beam may be parallel, fan, cone-beam or pencil. The detector module provides models for some phosphor screens for phosphor-based detectors (e.g. CsI, $\mathrm{Gd}_{2} \mathrm{O}_{2} \mathrm{~S}$ ), coupled with charge couple devices. These models include absorption of x-rays in the phosphor screen and a subsequent production of light quanta as well as the use of the Modulation Transfer Function for the corresponding detector.

X-ray projection images are obtained with simulation of x-ray photon transport starting from the x-ray source, passing through the breast model and reaching the detector. Figure 3 depicts the acquisition geometry that is used for mammography simulation.

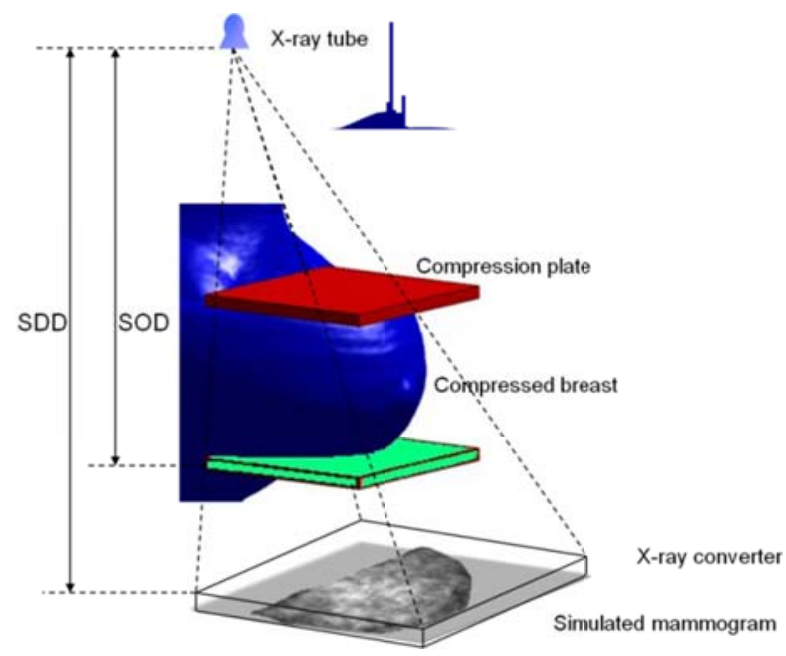

Figure 3. Simulation of x-ray mammography. SOD and SDD are source to object and source to detector distances.

Main parameters of the geometry are the source to detector and object to detector distances, the acquisition arc length and the number of projection images. Siddon's algorithm for tracing the x-rays from the source to detector pixels is applied ${ }^{[25]}$. The source emits a number $\mathrm{N}_{0}$ of $\mathrm{x}$ rays with energy sampled from the incident energy distribution $\mathrm{E}_{0}$ towards each pixel of the detector. The attenuation due to the breast tissues distributed in the voxelized breast phantom is calculated by adding the contributions of each voxel. The transmitted energy E reaching the detector pixel is calculated using Lambert-Beer's law:

$$
E=\sum_{j=E_{\min }}^{E_{\max }} E_{j} N_{j} \cdot \exp \left(-\sum_{i=1}^{n} \mu_{i} d_{i}\right)
$$

where $\left[E_{\min }: E_{\max }\right]$ is the energy range of the incident $x$-rays at the $x$-ray source, $n$ is the number of regions (voxels) in the breast phantom through which the x-ray crosses, while the parameters $\mu_{i}$ and $d_{i}$ are the attenuation coefficient and the path length through each of the voxels encountered, respectively.

To calculate the incident photon fluence, we exploit the relationship between the incident air kerma, $K$, measured at the upper surface of the breast phantom and the photon fluence: 


$$
K_{c, a i r}=\left[\frac{\mu_{e n}}{\rho}\right]_{a i r} \cdot E \cdot \Phi
$$

where $K_{\mathrm{c}, \text { air }}$ is the incident air kerma, $E$ is the energy of the incident photons, $\Phi$ is the photon fluence and $\left[\mu_{\mathrm{en}} / \rho\right]_{\text {air }}$ is the mass energy absorption coefficients for air, taken from Hubbell and Seltzer ${ }^{[26]}$. The air kerma is derived from the relationship with the mean glandular dose (MGD):

$$
M G D=K \cdot g \cdot c \cdot s \quad[\mathrm{mGy}]
$$

In this expression, $\mathrm{g}, \mathrm{c}$ and $\mathrm{s}$ are conversion factors depending on factors like breast thickness, glandularity and incident spectra. Their values were taken from the literature ${ }^{[13,27]}$. The MGD values in mGy are defined according to the European guidelines for quality assurance in breast cancer screening and diagnosis for a standard unilateral mammography ${ }^{[28]}$.

Expression 1 shows that calculated projection images represent 2D energy or photon distributions (depending on the detector type) of the radiation reaching the detector. Images obtained with this approach are called analytical or free of scatter, since they exploit the analytical relationships for x-ray matter interaction. Poisson quantum noise is also added to the original ideal images, using a Gaussian random number generator, with a variance set equal to the number of photons that are incident on each detector pixel.

\subsubsection{Visualization module}

There are several utilities that are used to visualize the synthetic projection images and 3D breast models. One utility is used to display the simulated mammographic projection images as shown in Figure 4a. Figure $4 \mathrm{~b}$ depicts the 3D visualization module with a breast model composed of duct trees, Cooper ligaments and lymphatic system. Display of 3D breast models is implemented with the help of the OpenGL library. For dual-energy applications, there is a dedicated module to perform dual-energy imaging based on projections acquired with two energies. In this case, a simple subtraction algorithm and weighting of the initial projections is applied.

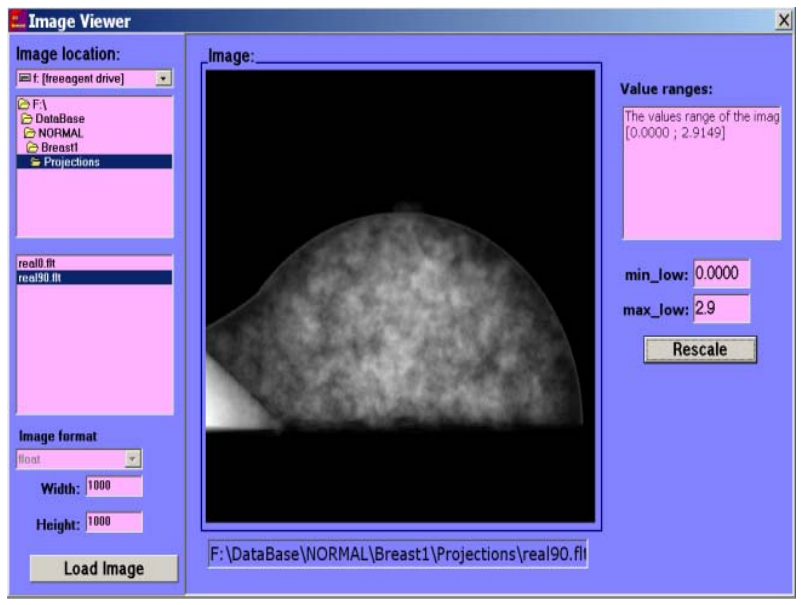

(a)

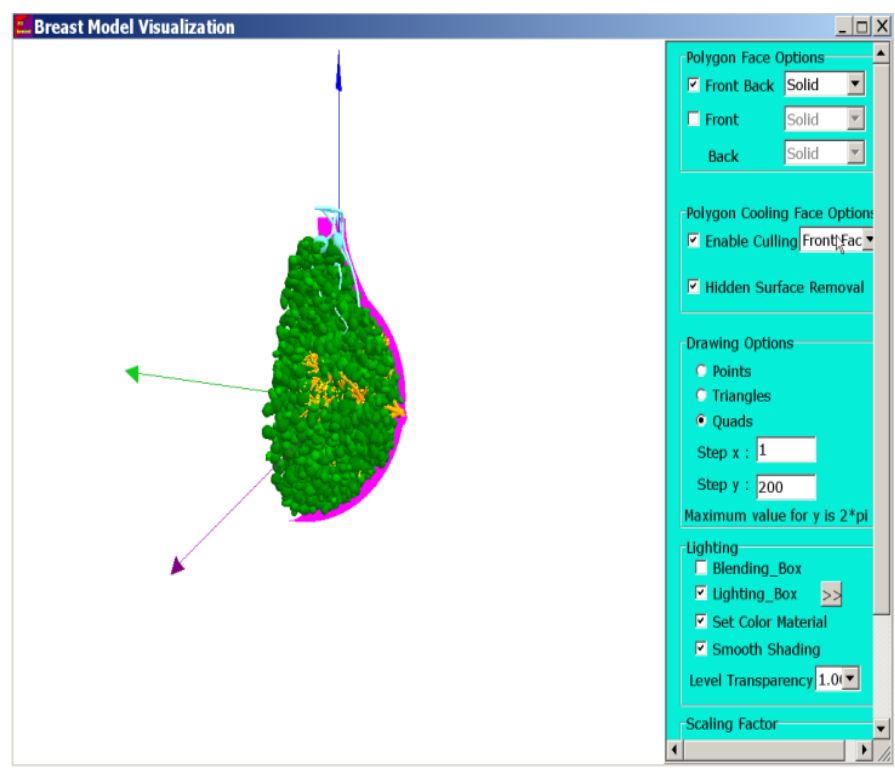

(b)

Figure 4. Visualization Module: screenshots from (a) mammography image display and (b) 3D visualization of generated breast models. 


\subsection{Computational issues}

The BreastSimulator software package can be run under Windows or Linux. For Windows, BreastSimulator is compiled with Borland $\mathrm{C}++$ Builder 6.0 as a single application with a graphical user interface (GUI) that combines all four modules. The executable for Windows, with relevant help and demo files, can be obtained for free, upon request, by any research team working in the field.

Although Borland C++ Builder 6.0 allowed for the development of a GUI under Windows, it can only create 32-bit code and hence the application is limited with respect to the size and resolution of the breast model and synthetic images that can be generated. To obtain higher resolution matrices and increased speed, the different modules of the package are compiled as 64-bit console applications and run under Linux. Still some parameter information is prepared using the Windows program.

The generation of largely 'realistic' breast phantoms is computationally intensive. Fortunately, the algorithm is highly subjected to parallelization. A possibility which we exploited is the use of the Message Passing Interface for interprocessor communication implemented through mpich2 ${ }^{[29]}$. Parallelization concerns the subroutines that are computationally intensive, such as the creation of the duct trees, the cooper ligaments, the background texture and the compression simulation. These processes are undertaken by the slave processors that read the broadcasted information, write the voxel value in a buffer and send the calculated data when requested by the master processor. In case of breast compression, the breast volume is divided into several sub-volumes and each of these sub-volumes is processed by a separate slave for each iteration step.

\section{Results: selected examples}

Here we demonstrate the use of the BreastSimulator platform for carrying out optimization work related to the choice of appropriate parameters for dual-energy mammography and dual-energy BT, studying the characteristics of scattered radiation from breast tissues, as well as its application with real breast images for compression purposes.

\subsection{Defining the best pair of monochromatic energies for 2D and 3D dual-energy breast imaging at synchrotron facilities}

The x-ray radiation produced within the storage ring of a synchrotron, such as Elettra in Trieste (Italy), is highly intense and monochromatic. Due to the long duration and the high cost of the experiments at these facilities as well as the limited time to perform these experiments, simulation work prior to real experimentation needs to be carried out. This is especially valid for the case of testing dual-energy mammography and dual-energy tomosynthesis at such a facility. Due to the large number of energy combinations for producing a dual-energy image, it is necessary to perform initial simulations that would define the optimal pair of 'low' and 'high' monochromatic x-ray energies for 2D and 3D dual-energy imaging.

To achieve this goal, we modeled the acquisition geometry at synchrotron facilities, the volumes used in irradiation and the $\mathrm{x}$-ray beam transport using BreastSimulator.

(a) Sample. A software phantom of a breast was generated using the methodology for breast model creation. The computed 3D uncompressed breast approximates a medium sized breast $\left(540 \mathrm{~cm}^{3}\right)$, with $50 \%$ adipose and $50 \%$ glandular tissue. The model also contained one calcification embedded in the heterogeneous 3D background, simulated as a calcium carbonate sphere with diameter of $0.6 \mathrm{~mm}$. Further, compression was applied to the breast model using the Compression Module that resulted in a compressed breast model with thickness of $6 \mathrm{~cm}$.

(b) Geometry. Projection images at synchrotron facilities are obtained using acquisition geometry (see Figure 5a) that differs from the conventional geometries utilized in mammography x-ray machines. At synchrotron facilities, the beam is 
laminar and located at a fixed position. The object that should be irradiated is mounted on a scanning stage. In order to acquire $2 \mathrm{D}$ images, the sample and the detector are scanned vertically and the scanning step determines the spatial resolution in the scanning direction.

For the purposes of this study, we simulated the geometry shown in Figure 5a using the approach depicted in Figure 5b. The source is represented by a plane source with spatial resolution that corresponds to the detector resolution. Source to object plane and source to detector distances were set to $13.09 \mathrm{~m}$ and $23 \mathrm{~m}$ respectively. The detector was modeled as a flat panel detector with a $\mathrm{Gd}_{2} \mathrm{O}_{2} \mathrm{~S}$ scintillation layer with thickness of $41 \mu \mathrm{m}$. Parallel x-rays emerging from the plane source were simulated through the phantom using analytical relationships for $\mathrm{x}$-ray matter interaction (expression 2).

(c) Simulations. The simulation study for determining the optimal pair of energies to be used with dual-energy involved the synthesis of 225 dual-energy images obtained from combinations of 'low' and 'high' energy images acquired in the energy range 14 to $28 \mathrm{keV}$. This energy range was defined given the energy limits at the SYRMEP beamline at Elettra, Trieste at which images with sufficient quality and reasonable scanning time may be acquired. The MGD to the breast for all combinations was limited to $5 \mathrm{mGy}$. The MGD proportions were set to $2.4 \mathrm{mGy}$ and $1.6 \mathrm{mGy}$ for 'low' and 'high' energy images respectively. The choice of low to high MGD ratio was based on our previous dual-energy simulation study ${ }^{[30]}$.

Initially, fifteen plane projection images of this phantom corresponding to specific incident $\mathrm{x}$-ray beam energy were simulated. These images represent the $2 \mathrm{D}$ distributions of the line integrals of the attenuation coefficient along the paths of the x-rays, registered at the detector surface. For each energy combination, the incident air kerma and therefore the incident photon fluence were calculated based on knowledge for the desired MGD, breast thickness and glandularity.

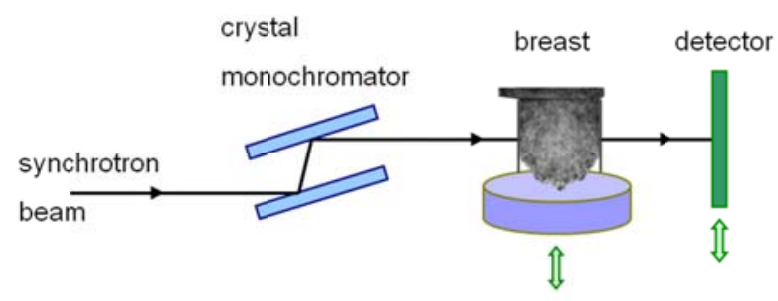

(a)

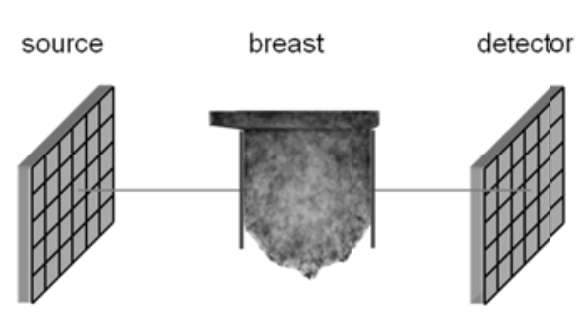

(b)

Figure 5. Irradiation geometry: (a) Beam setup at synchrotron facility. The laminar beam passes through a crystal monochromator that is adjusted to the desired photon energy. Sample is placed on a platform that can move and rotate in order to produce projection images at any angle. (b) Simulated acquisition geometry.

Dual-energy images were obtained based on a linear subtraction algorithm ${ }^{[31]}$. The weighting coefficients, the $R$ values, for each image combination were calculated. The figure of merit (FOM) used to determine the optimal pair of energies was the Signal-to-Noise Ratio (SNR) measured in the area of the projected calcification. The SNR was calculated as $m_{\text {feature }} / \sigma_{\mathrm{bg}}$, where $m_{\text {feature }}$ is the mean pixel value of the calcification and $\sigma_{\mathrm{bg}}$ is the standard deviation of pixel values in the background region chosen to be a square area of 1110 pixels adjacent to the calcification.

Three-dimensional distribution of the FOM for the $0.6 \mathrm{~mm}$ calcification is shown as a function of low and high incident energies in Figure 6. Analysis of the FOM results showed an optimum low energy interval $17 \pm \sigma_{\mathrm{L}} \mathrm{keV}$ and a high energy interval $28 \pm \sigma_{\mathrm{H}} \mathrm{keV}$, where $\sigma_{\mathrm{L}}$ and $\sigma_{\mathrm{H}}$ correspond to the $5 \%$ margin for FOM. The R value for this specific combination was 0.5 . Figure $6 \mathrm{~b}, 6 \mathrm{c}$ depicts the comparison of a projection image taken at $17 \mathrm{keV}$ and a dual-energy image calculated from images with 17 and $28 \mathrm{keV}$. The improved contrast of the calcification in the dual-energy image compared to the 
normal mammographic image can be readily seen. These two energies are currently used in a study related to dual-energy BT.

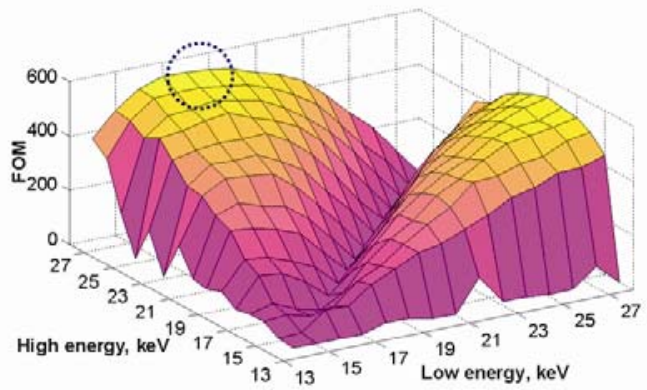

(a)

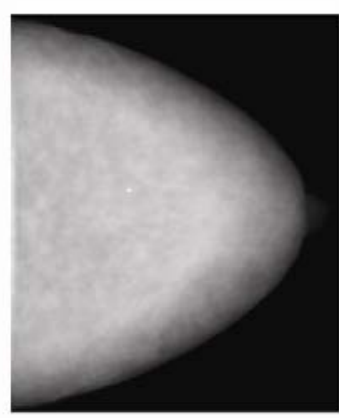

(b)

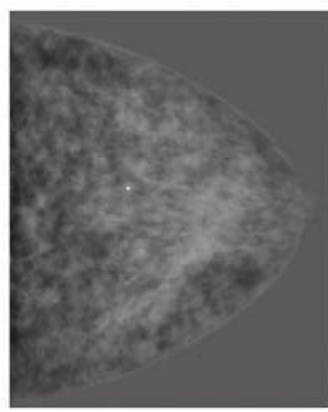

(c)

Figure 6. Dual-energy study: (a) FOM distribution for a calcification with a size of $0.6 \mathrm{~mm}$ detected with $\mathrm{Gd}_{2} \mathrm{O}_{2} \mathrm{~S}_{\text {based detector; }}$ (b) Planar projection image simulated with $17 \mathrm{keV}$ and dose equivalent to the dose for dual-energy image; (c) dual-energy image obtained from images acquired with 17 (low) and 28 (high) keV.

\subsection{I nvestigating compression with breast models from clinical data}

Breast compression is an essential part of mammography and is an important component in producing high quality mammographic images. Breast compression allows for dose and scatter reduction, decreased motion and geometrical unsharpness, increased contrast, and the separation of breast structures. Moreover, the use of compression results in a more homogeneous thickness across the breast, providing fairly uniform density over the mammogram.

The following example demonstrates the use of a module from BreastSimulator for studying the effect of compression on soft tissues. Breast models were created from slices, obtained from breast CT scans of breasts of different sizes and compositions at the Department of Radiology and Imaging Sciences at Emory University, USA. Initially, the data were de-noised and segmented in order to obtain compositional breast models, i.e. models composed of skin, glandular and adipose tissues ${ }^{[23]}$. An example is shown in Figure $7 \mathrm{a}, 7 \mathrm{~b}$ where the uncompressed breast in 3D and a slice from it are shown. The voxel size of the 3D breast model was $0.28 \mathrm{~mm}$ in each direction, while the whole breast volume was calculated to be $2160 \mathrm{~cm}^{3}$. This volume was subjected to the compression algorithm to create a compressed version of the breast. The compression plates were placed at two different positions to generate breasts with $5.8 \mathrm{~cm}$ and $7.0 \mathrm{~cm}$ compressed thickness. A slice from the compressed version is depicted in Figure 7c.

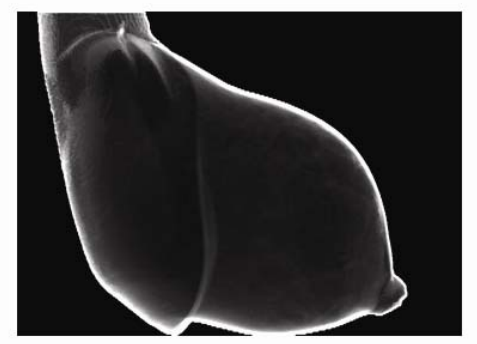

(a)

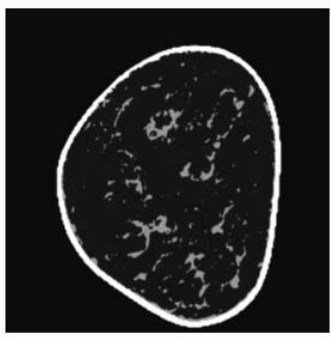

(b)

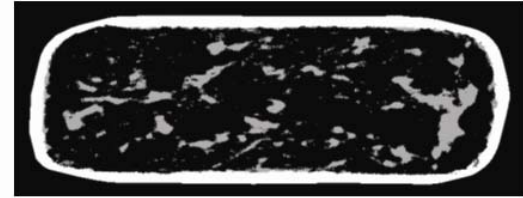

(c)

Figure 7. Compression with breast models from clinical data. (a) Anthropomorphic breast model created from slices from breast CT; (b) Slice from the uncompressed breast model; (c) $\mathrm{S}$ from the compressed version at $5.8 \mathrm{~cm}$. 
Further, to simulate mammography, we generated projection images in the cranio-caudal view. For this purpose, we used the same modeled $\mathrm{Gd}_{2} \mathrm{O}_{2} \mathrm{~S}$ detector from the previous example. The generated images have a size of $4000 \times 4000$ pixels with a pixel pitch of $0.1 \mathrm{~mm}$. Comparison of projection images simulated from uncompressed and compressed breasts are shown in Figure $8 \mathrm{a}, \mathrm{b}$, c. The total dose to the breast was set to $2.5 \mathrm{mGy}$. Using expressions 2 and 3, we calculated the initial photon fluence for energy of $17 \mathrm{keV}$.

The visual comparison shows that photons are not reaching some parts of the detector in case of the uncompressed breast. This was expected since the same value of MGD (and therefore the initial photon fluence) was used for both the compressed and uncompressed breast conditions. In the uncompressed state, the breast is thicker, resulting in higher attenuation, and therefore fewer photons reach the detector. This result in poor visual appearance when compared to the image from the compressed breasts (see Figure $8 \mathrm{~b}$ and $8 \mathrm{c}$ ). To compare the contrast improvement, we calculated the SNR in the three projection images. The calculated SNR was about 5 and 4.3 times higher in images obtained from compressed breast with thickness of $5.8 \mathrm{~cm}$ and $7.0 \mathrm{~cm}$, respectively compared to the uncompressed breast, mainly due to the reduced thickness.

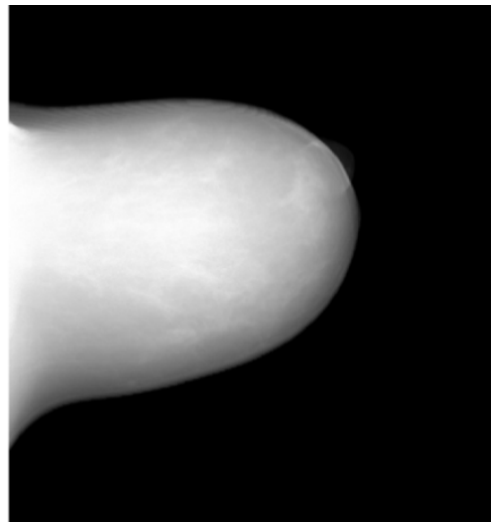

(a)

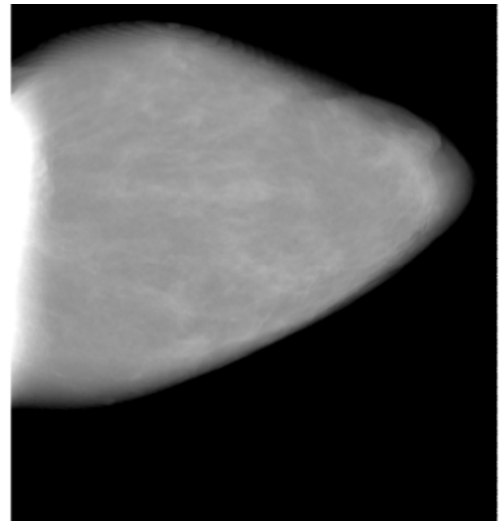

(b)

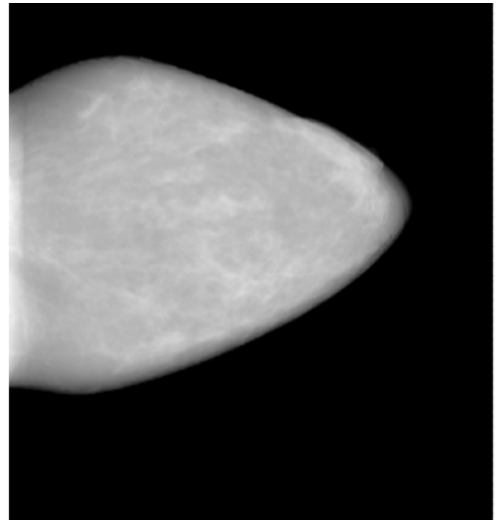

(c)

Figure 8. Simulated mammographic images of (a) the uncompressed breast shown in Figure 7a and its compressed versions with a thickness of $5.8 \mathrm{~cm}$ (b) and $7.0 \mathrm{~cm}$ (c). The MGD is $2.5 \mathrm{mGy}$.

\subsection{I nvestigating the energy and angular distribution of beams that exit the breast in mammography}

Projection images obtained from the BreastSimulator are produced very quickly due to the use of an analytical x-ray tracing model. A more complex level of simulation of the radiation interaction at the absorber and the detector includes the generation of $\mathrm{x}$-rays from the $\mathrm{x}$-ray source, simulation of scattered radiation and the determination of the energy absorption, at both the detector and the absorber. This is achieved by the use of Monte Carlo techniques. Monte Carlo codes used with breast models with different composition may be exploited effectively in the evaluation of novel x-ray sources or detectors as well as to study the influence of the breast composition and thickness on the transmitted x-ray beam and therefore on the quality of the generated mammogram. An example is demonstrated below.

A small dense breast model $\left(134 \mathrm{~cm}^{3}\right)$ with no lesion included was designed using the Breast Modeling Module. The model was compressed in the Compression Module to a thickness of $40 \mathrm{~mm}$. Mammographic images were simulated using a Monte Carlo x-ray simulation software package developed in-house ${ }^{[32]}$. The acquisition geometry, shown in Figure 3, includes source-detector and source-breast distances equal to $650 \mathrm{~mm}$ and $600 \mathrm{~mm}$, respectively. The images are 
synthesized with two mammographic spectra: $28 \mathrm{kV} \mathrm{Mo,} 0.030 \mathrm{~mm}$ Mo and $32 \mathrm{kV} \mathrm{W}, 0.050 \mathrm{~mm} \mathrm{Rh}{ }^{[27,33]}$. Incident exposure was set to $7 \mathrm{mGy}$.

Figure 9a, b shows the energy distribution of x-rays transmitted through the breast phantom for the Mo/Mo and W/Rh spectra, respectively. The mean energy of the x-rays that exit the breast phantom in case of W anode is slightly higher than the mean energy of the x-rays that reach the detector in case of Mo anode. The angular distribution of the x-rays that exit the breast phantom is depicted in Figure 9c for the two incident spectra. This figure shows the relative number of scattered photons per unit solid angle as a function of the exit angle. For both spectra, the angular distribution may be approximated with bell-shaped curves. The mean exit angles were $23^{\circ}$ for $\mathrm{W} / \mathrm{Rh}$ and $22^{\circ}$ for $\mathrm{Mo} / \mathrm{Mo}$ incident spectra. The increase in the incident energy results in an increase in the mean exit angle. This is attributed to the increasing probability of multiple scattering events in the breast resulting in more isotropic angular distribution ${ }^{[34]}$.

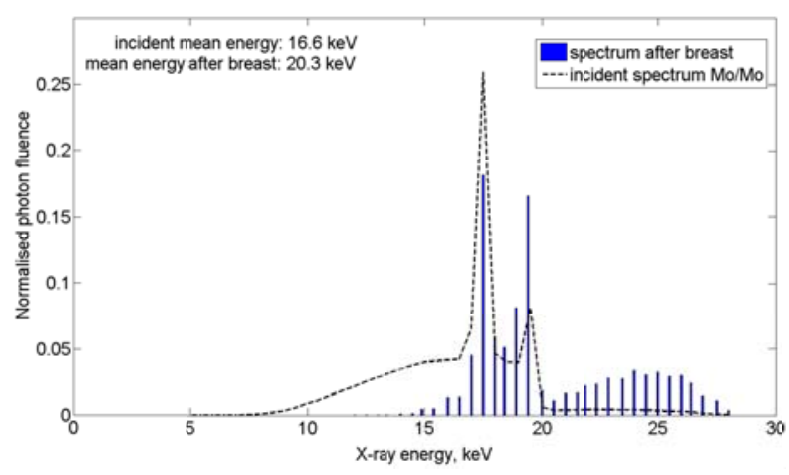

(a)

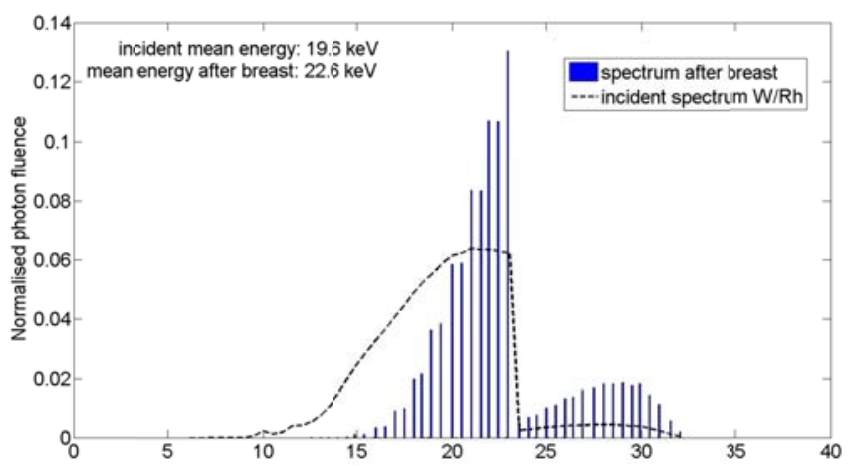

(b)

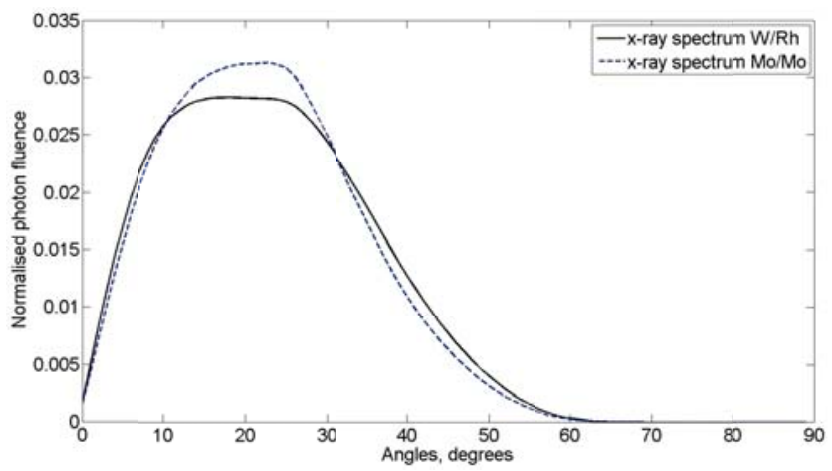

Figure 9. Energy and angular distributions of x-rays exiting the breast models: $(a, b)$ energy distributions of photons incident and exiting the dense breast for $\mathrm{Mo} / \mathrm{Mo}$ and $\mathrm{W} / \mathrm{Rh}$ incident spectra; (c) angular distribution of scattered photons exiting the breast for the two incident $\mathrm{x}$-ray spectra.

(c)

The above distributions are further used with anti-scattered grid and image recorder configurations in studies that aim to evaluate various detector performances applied for mammography.

\section{Conclusions}

Computer modeling and simulation offers a cost effective, practical and flexible approach, compared to clinical trials, to understand and optimize the performance of new technologies, provided these tools are validated and include the necessary patient related aspects. For investigations in the field of x-ray imaging of the breast, there is a need to develop models of the breast that possess sufficient realism, are easy to understand and manipulate by any user, and can be modified for the specific aims of the research studies. 
In this paper we presented the software package BreastSimulator, a research tool for performing investigations in breast imaging techniques and demonstrated some of its basic functionalities to carry out $\mathrm{x}$-ray breast imaging studies. We believe that it is a valuable tool, and based on its functionality it will contribute considerably to the development, testing and optimization of novel breast imaging techniques, the evaluation of the effectiveness of the proposed new modalities to improve cancer detection and the estimation of the needed material, time and financial resources for their eventual implementation in practice.

\section{References}

[1] EUROSTAT. Causes of death in the EU. 2006; 12. http://epp.eurostat.ec.europa.eu/cache/ITY_OFFPUB/KS-NK-06-010/EN/KS-NK-06-010-EN.PDF

[2] Ferlay J, Autier P, Boniol M, Heanue M, Colombet M, Boyle P. Estimates of the cancer incidence and mortality in Europe in 2006. Ann Oncol. 2007; 18: 581-592.

[3] Durfee S, Selland D, Smith D, Lester S, Kaelin C, Meyer J. Sonographic evaluation of clinically palpable breast cancers invisible on mammography. Breast J. 2000; 6: 5. http://onlinelibrary.wiley.com/doi/10.1046/j.1524-4741.2000.99111.x/pdf

[4] Poplack S P, Tosteson T D, Kogel C A, Nagy H M. Digital breast tomosynthesis: Initial experience in 98 women with abnormal digital screening mammography. AJR Am J Roentgenol. 2007; 3: 8. http:/www.ajronline.org/content/189/3/616.full.pdf

[5] Gur D, Abrams G S, Chough D M, Ganott M A, Hakim C M, Perrin R L, et al. Digital breast tomosynthesis: observer performance study. AJR Am J Roentgenol. 2009; 193: 586-591. http://www.ncbi.nlm.nih.gov/pubmed/19620460

[6] Karellas A,Vedantham S. Breast cancer imaging: a perspective for the next decade. Med Phys. 2008; 35: $4878-4897$. http://www.ncbi.nlm.nih.gov/pubmed/19070222

[7] Highnam R, Brady M, Shepstone B. A representation for mammographic image processing. Med Image Anal. 1996 ; 1 : 1-18. http://www.ncbi.nlm.nih.gov/pubmed/9873918

[8] Bakic P R, Zhang C, Maidment A D. Development and characterization of an anthropomorphic breast software phantom based upon region-growing algorithm. Med Phys. 2011; 38: 3165-3176. http://www.ncbi.nlm.nih.gov/pubmed/21815391

[9] Hunt R A, Dance D R, Bakic P R, Maidment A D, Sandborg M, Ullman G, et al. Calculation of the properties of digital mammograms using a computer simulation. Radiat Prot Dosimetry. 2005; 114: 395-398.

http://www.ncbi.nlm.nih.gov/pubmed/15933144

[10] Bliznakova K, Suryanarayanan S, Karellas A, Pallikarakis N. Evaluation of an improved algorithm for producing realistic 3D breast software phantoms: application for mammography. Med Phys. 2010; 37: 5604-5617. http://www.ncbi.nlm.nih.gov/pubmed/21158272

[11] Peplow D E, Verghese K. Digital mammography image simulation using Monte Carlo. Med Phys. 2000; $27: 568-579$. http://www.ncbi.nlm.nih.gov/pubmed/10757608

[12] Sechopoulos I, Suryanarayanan S, Vedantham S, D'Orsi C, Karellas A. Computation of the glandular radiation dose in digital tomosynthesis of the breast. Med Phys. 2007; 34: 221-232. http://www.ncbi.nlm.nih.gov/pubmed/17278508

[13] Dance D R. Monte Carlo calculation of conversion factors for the estimation of mean glandular breast dose. Phys Med Biol. 1990; 35: 1211-1219. http://www.ncbi.nlm.nih.gov/pubmed/2236205

[14] Thacker S C, Glick S J. Normalized glandular dose (DgN) coefficients for flat-panel CT breast imaging. Phys Med Biol. 2004; 49: 5433-5444. http://www.ncbi.nlm.nih.gov/pubmed/15724534

[15] Sechopoulos I, Feng S S, D'Orsi C J. Dosimetric characterization of a dedicated breast computed tomography clinical prototype. Med Phys. 2010; 37: 4110-4120. http://www.ncbi.nlm.nih.gov/pubmed/20879571

[16] Chen B, Shorey J, Saunders R S, Jr., Richard S, Thompson J, Nolte L W, et al. An anthropomorphic breast model for breast imaging simulation and optimization. Acad Radiol. 2011; 18: 536-546. http://www.ncbi.nlm.nih.gov/pubmed/21397528

[17] Ma A K, Gunn S, Darambara D G. Introducing DeBRa: a detailed breast model for radiological studies. Phys Med Biol. 2009; 54: 4533-4545. http://www.ncbi.nlm.nih.gov/pubmed/19556683

[18] Bliznakova K, Bliznakov Z, Bravou V, Kolitsi Z, Pallikarakis N. A three-dimensional breast software phantom for mammography simulation. Phys Med Biol. 2003; 48: 3699-3719. http://www.ncbi.nlm.nih.gov/pubmed/14680268

[19] Dance D R, Hunt R A, Bakic P R, Maidment A D, Sandborg M, Ullman G, et al. Breast dosimetry using high-resolution voxel phantoms. Radiat Prot Dosimetry. 2005; 114: 359-363. http://www.ncbi.nlm.nih.gov/pubmed/15933137

[20] Kontos D, Ikejimba L C, Bakic P R, Troxel A B, Conant E F, Maidment A D. Analysis of parenchymal texture with digital breast tomosynthesis: comparison with digital mammography and implications for cancer risk assessment. Radiology. 2011; 261 : 80-91. http://www.ncbi.nlm.nih.gov/pubmed/21771961 
[21] Huang S Y, Boone J M, Yang K, Packard N J, McKenney S E, Prionas N D, et al. The characterization of breast anatomical metrics using dedicated breast CT. Med Phys. 2011; 38: 2180-2191. http://www.ncbi.nlm.nih.gov/pubmed/21626952

[22] Li C M, Segars W P, Tourassi G D, Boone J M, Dobbins J T, 3rd. Methodology for generating a 3D computerized breast phantom from empirical data. Med Phys. 2009; 36: 3122-3131. http://www.ncbi.nlm.nih.gov/pubmed/19673211

[23] Yang X, Sechopoulos I, Fei B. Automatic tissue classification for high-resolution breast CT images based on bilateral filtering. In Proceedings of the SPIE: 2011; Lake Buena Vista, Florida, USA Edited by B. Dawant and D. Haynor. http://spiedigitallibrary.org/proceedings/resource/2/psisdg/7962/1/79623H_1?isAuthorized=no

[24] Zyganitidis C, Bliznakova K, Pallikarakis N. A novel simulation algorithm for soft tissue compression. Med Biol Eng Comput. 2007; 45:661-669. http://www.ncbi.nlm.nih.gov/pubmed/17551761

[25] Siddon R L. Fast calculation of the exact radiological path for a three-dimensional CT array. Med Phys. 1985; $12: 252-255$. http://www.ncbi.nlm.nih.gov/pubmed/4000088

[26] Hubbell J H, Seltzer S M. Tables of x-ray mass attenuation coefficients and mass energy-absorption coefficients $1 \mathrm{keV}$ to $20 \mathrm{MeV}$ for elements $Z=1$ to 92 and 48 additional substances of dosimetric interest, 1995.

[27] Dance D R, Skinner C L, Young K C, Beckett J R,Kotre C J. Additional factors for the estimation of mean glandular breast dose using the UK mammography dosimetry protocol. Phys Med Biol. 2000; 45: 3225-3240. http://www.ncbi.nlm.nih.gov/pubmed/11098900

[28] Perry N, Broeders M, Wolf C, Törnberg S, Holland. R, Karsa L. European guidelines for quality assurance in breast cancer screening and diagnosis. 4th ed. (Office for Official Publications of the European Communities, Luxembourg, 2006). $2006 ; 416$. http://www.euref.org/european-guidelines

[29] MPICH2. http://www-unix.mcs.anl.gov/mpi/mpich2/ (November 2011 date last accessed).

[30] Bliznakova K, Kolitsi Z, Pallikarakis N. Dual-energy mammography: simulation studies. Phys Med Biol. 2006; 51: 4497-4515. http://www.ncbi.nlm.nih.gov/pubmed/16953039

[31] Boone J M. Color mammography. Image generation and receiver operating characteristic evaluation. Invest Radiol. 1991; 26: 521-527. http://www.ncbi.nlm.nih.gov/pubmed/1860758

[32] Lazos D, Bliznakova K, Kolitsi Z, Pallikarakis N. An integrated research tool for X-ray imaging simulation. Comput Methods Programs Biomed 2003;70:241-251. http://www.ncbi.nlm.nih.gov/pubmed/12581556

[33] Fahrig R, Yaffe M J. Optimization of spectral shape in digital mammography: dependence on anode material, breast thickness, and lesion type. Med Phys. 1994; 21: 1473-1481. http://www.ncbi.nlm.nih.gov/pubmed/7838059

[34] Chan H P, Doi K. Physical characteristics of scattered radiation in diagnostic radiology: Monte Carlo simulation studies. Med Phys. 1985; 12: 152-165. http://www.ncbi.nlm.nih.gov/pubmed/4000070

[35] Kellner A L, Nelson T R, Cervino L I,Boone J M. Simulation of mechanical compression of breast tissue. IEEE Trans Biomed Eng. 2007; 54: 1885-1891. http://www.ncbi.nlm.nih.gov/pubmed/17926687

[36] Samani A, Zubovits J, Plewes D. Elastic moduli of normal and pathological human breast tissues: an inversion-technique-based investigation of 169 samples. Phys Med Biol. 2007; 52: 1565-1576. http://www.ncbi.nlm.nih.gov/pubmed/17327649 